\title{
Quinua de valle (Chenopodium quinoa Willd.): fuente valiosa de resistencia genética al mildiu (Peronospora farinosa Willd.)
}

\section{Quinoa from Valley (Chenopodium quinoa Willd.): Valuable source of genetic resistance to powdery mildew (Peronospora farinosa Willd.)}

\author{
Gabriel Julio $^{1 *}$, Luna Nayra ${ }^{2}$, Vargas Amalia ${ }^{3}$, Magne Jury ${ }^{1}$, Angulo Ada ${ }^{1}$, La Torre Jaime², Bonifacio Alejandro ${ }^{3}$.
}

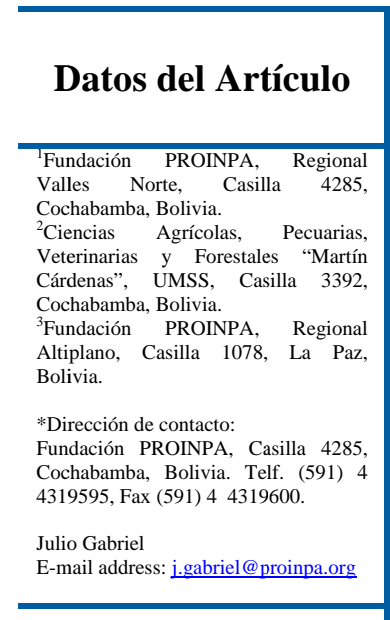

\section{Palabras clave: \\ Grano, \\ cultivares, \\ Peronospora farinosa relativa, \\ rendimiento, \\ diámetro de semilla. \\ J Selva Andina Res Soc 2012; 3(2):27-44. \\ Historial del artículo \\ Recibido Septiembre, 2012. Devuelto Diciembre 2012 \\ Aceptado Enero, 2013. \\ Disponible en línea, Febrero, 2013. \\ Key words: \\ Grain,
cultivars, \\ Peronospora farinosa relative, \\ yield, seed diameter.}

\section{Editado por:}

"Selva Andina

Research

Society”

\section{Resumen}

Con el objetivo de identificar cultivares de quinua resistentes a mildiu (Peronospora farinosa) de alto rendimiento y tamaño grande de grano, se evaluó 36 cultivares bajo dos tipos de control para mildiu y un testigo en el valle bajo de Cochabamba. Se determinó el Area Bajo la Curva de Progreso de Peronsopora farinosa relativa (ABCPPFrel), el rendimiento y otras 11 variables cuantitativas. Los resultados mostraron que los cultivares 01Tardía, 08Tardía, 12Tardía, 04Tardía, 11Tardía 10Tardía, 19Tardía y 18Tardía fueron susceptibles y los cultivares H172, A26, A03, A16, A22, A14 y H171 fueron los más resistentes. Los cultivares A40, H177, A26, H172, A25, A1 y H176 mostraron rendimientos entre 3.4 a $6.34 \mathrm{t} \mathrm{ha}^{-1}$. Los cultivares 15Tadía, 03 Tardía, 14Tardía, H173, H171, A25, H176 y H172 con la estrategia de control químico y con el tricobal reaccionaron favorablemente contra el mildiu, lo cual fue asociado a los niveles de resistencia en cada cultivar. Finalmente, hubo una alta correlación negativa y significativa entre el ABCPPFrel y las variables madurez fisiológica, longitud de planta, longitud de panoja, diámetro de tallo, diámetro de panoja y peso de 100 semillas. Esto mostró que cuando el ataque del mildiu es severo, afecta también al rendimiento en grano.

2012. Journal of the Selva Andina Research Society. Bolivia. Todos los derechos reservados.

\section{Abstract}

In order to identify quinoa resistant cultivars to powdery mildew (Peronospora farinosa) of high yield and large grain size, 36 cultivars were evaluated under two different fungicide ap-plications and a control in the Valle Bajo from Cochabamba. We determined the realtive Area under Develop Progress Curve of Peronospora farinosa (AUDPCPF relative), the yield and another eleven quantitative variables. The results showed that cultivars 01Tardía, 08Tardía, 12Tardía, 04Tardía, 11Tardía 10Tardía, 19Tardía y 18Tardía were susceptible and the cultivars H172, A26, A03, A16, A22, A14 and H171 were resistant. The cultivars A40, H177, A26, H172, A25, A1 y H176 showed yields from 3.4 to $6.34 \mathrm{t} \mathrm{ha}^{-1}$ ). The cultivars 15 Tardía, 03 Tardía, 14 Tardía, H173, H171, A25, H176 and H172 with chemical control strategy and the tricobal reacted favorably against powdery mildew, which was associated with levels of resistance in each cultivar. Finally, there was a high significant negative correlation between the variables AUDPCPF relative and physiological maturity, plant length, panicle length, stem diameter, panicle diameter and weight of 100 seeds. This showed that when the attack of mildew is severe, also affects the grains yield.

ㄷ 2012. Journal of the Selva Andina Research Society. Bolivian. All rights reserved. 


\section{Introducción}

La quinua (Chenopodium quinoa Willd.) es un cultivo nativo de la región Andina. Estas zonas son consideradas como uno de los ocho centros de origen de las especies cultivadas de quinua. La mayoría de los investigadores coinciden en indicar que la quinua es originaria del altiplano que comparten Perú y Bolivia, ya que en dichas áreas se encuentra la mayor diversidad de plantas cultivadas y parientes silvestres (Gandarillas 1979, Muñoz et al 1990). El cultivo de la quinua es importante por sus altos valores nutricionales, con un contenido de proteína promedio de 17\% (López 1976). Debido al elevado y balanceado contenido de aminoácidos esenciales de su proteína, la quinua es considerada como uno de los alimentos del reino vegetal que provee todos los aminoácidos esenciales, que se encuentran cerca de los estándares de nutrición humana establecidos por la FAO (FAO 2011).

A nivel mundial, la demanda de quinua por su alto valor nutricional alcanza a $70000 \mathrm{t}^{\mathrm{año}} \mathrm{O}^{-1}$. Esta demanda de quinua es sostenida por más de 51 países, donde el 90\% está producido en la región Andina (FAO 2011). Los principales productores de este grano a nivel internacional son: Bolivia, Perú, Ecuador, y Colombia. Bolivia cuenta con más de 47534 ha cultivadas y alrededor de $21900 \mathrm{t}$ cosechadas (FAO 2011), de las cuales el $49 \%$ es consumida por las familias productoras, el $35 \%$ se vende en los mercados locales y el resto (3500 t) es para los mercados externos, constituyéndose así como el primer productor y exportador de quinua en el mundo (Viñas 2000).
En Bolivia se dispone de una colección de germoplasma de quinua conformada por 3121 accesiones que se conservan en el Banco Nacional de Germoplasma de Granos Altoandinos (BNGA). Esta amplia variabilidad genética comprende quinuas de gran parte de la región Andina, desde el Ecuador hasta el noroeste de Argentina, así como también quinuas de nivel de mar cultivadas en Chile y países de Europa. Esta amplia variabilidad que incluye cultivares avanzados, cultivares locales, especies silvestre y formas regresivas, constituye una relevante contribución a la estabilidad del cultivo en el país y en gran parte de la región Andina (Rojas 1998). El BNGA actualmente está bajo la custodia del Instituto Nacional de Innovación Agropecuaria y Forestal (INIAF) (Rojas et al 2010).

En Cochabamba, la producción del cultivo de quinua se distribuye principalmente en las zonas de las provincias de Quillacollo, Punata, Tiraque, con un rendimiento de 0.5 a $0.7 \mathrm{t} \mathrm{ha}^{-1}$. (IBTA 1987).

Sin embargo, la producción de quinua es afectada por diversos factores abióticos (heladas, sequia, granizada) y bióticos (plagas y enfermedades). Ortiz (1998), menciona que entre las plagas que causan daño en las quinuas están los cortadores de plantas tiernas (Feltia experta), minadores y destructores del grano (Scrobipalpula spp.), picadores y chupadores (Franklieniella spp.) y masticadores y defoliadores (Epicauta latitarsis). Entre las enfermedades una de las más importantes es el mildiu causado por el oomycete Peronospora 
farinosa F. sp. Chenopodii, que en lugares donde hay alta humedad relativa y temperaturas entre 12 a $22^{\circ} \mathrm{C}$, puede causar grandes pérdidas. Además de que su control es costoso, afecta a la salud del hombre y causa contaminación ambiental. Alandia et al (1979), Danielsen \& Ames (2000) encontraron que el mildiu bajo condiciones de alta presión de la enfermedad redujo los rendimientos de 33 a 58\% en varios cultivares de quinua (LP4B, La Molina 89, Blanca de Juli, Kancolla, Jujuy, Amarilla de Maranganí e Ingapirca). Utusaya, cultivar del altiplano Sur de Bolivia, fue el más afectado con una pérdida de 99\%. La enfermedad bajo condiciones de temperatura $\mathrm{y}$ humedad adecuadas para su desarrollo puede causar pérdidas del 100\% (Alandia 1979, Otazú et al 1976, Ochoa \& Danial 1999).

Se conoce que la mayoría de los cultivares de quinua del altiplano son susceptibles a la enfermedad (mildiu). Sin embargo, los cultivares de quinua de los valles que son sembrados por los agricultores como cultivos de contorno en sus parcelas, tienen resistencia variable a la enfermedad (mildiu), sobre las cuales poco o nada se conoce (Mujica 1988).

Con el fin de controlar a la enfermedad, los agricultores han utilizado tradicionalmente fungicidas en base a metalaxil. Sin embargo, los fungicidas son costosos, requieren múltiples aplicaciones, y finalmente, pueden ser superados por razas resistentes, ya que el patógeno es sexualmente recombinante (Aegerter et al 2002, Thomma 2003). Además, incrementan los costos de producción y afectan a la salud de los agricultores y al medioambiente (Gabriel 2010).
Para desarrollar cultivares resistentes que ayude al control de la enfermedad, existe una gran diversidad genética de cultivares de quinua que exhiben diversos grados de resistencia (Mujica 1988). Por ejemplo, los cultivares de valle que crecen en regiones donde la humedad es alta y la enfermedad está muy extendida, a menudo muestran resistencia alta a moderada al mildiu; mientras que en el sur del altiplano, los cultivares que crecen en estas regiones más secas muestran una mayor susceptibilidad (Alfano \& Collmer 1997, Tapia 1979). Si se dispusiera de cultivares resistentes se podría reducir el número de aplicaciones de fungicidas y lograr mejores rendimientos. $\mathrm{Al}$ mismo tiempo, la fertilización es importante para que las plantas sean más vigorosas y puedan resistir el ataque de enfermedades.

Por otra parte, Gabriel (2010) sugiere que la mejora genética de cultivares en función de su adaptación a nichos particulares tiene mayores ventajas y oportunidades de adopción, que el desarrollo de un único cultivar para varios ambientes, por lo que la estrategia debería estar dirigida a generar cultivares para nichos particulares.

\section{Materiales y métodos}

La investigación se realizó en el año agrícola 2010-2011 en predios de la Fundación PROINPA en la zona de El Paso a $15 \mathrm{~km}$ de la ciudad de Cochabamba en la provincia de Quillacollo, ubicado a $17^{\circ} 18^{\prime}$ de latitud Sud y $66^{\circ} 14^{\prime}$ de longitud Oeste, a una altitud de $2540 \mathrm{msnm}$. El clima es templado, la precipitación media anual es de $560.9 \mathrm{~mm}$ de la cual el $80 \%$ se distribuye entre los meses de diciembre a marzo, la temperatura 
media anual es de $18^{\circ} \mathrm{C}$, con una máxima $38.6^{\circ} \mathrm{C}$ y una mínima $9.3^{\circ} \mathrm{C}$, la humedad relativa media anual es de 64\% (SENAMHI 2010).

Se utilizó 36 cultivares de quinua para valle. De estos, 16 cultivares fueron proporcionadas por el programa de mejoramiento genético de PROINPA de la regional Altiplano de La Paz. Los restantes 20 cultivares fueron proporcionados por regional Valles Norte de PROINPA, Cochabamba.

La parcela se estableció en un diseño experimental de Bloques Completos al Azar en arreglo de Parcelas Divididas con cuatro repeticiones. Las repeticiones fueron sembradas escalonadamente cada siete días, para bloquear el efecto de época y así evitar la posible variación de las condiciones ambientales (Martinez-Garza 1988). Los cultivares en cada repetición fueron distribuidos aleatoriamente.

Los tratamientos fueron la combinación de dos factores: Factor A: 36 cultivares de quinua y Factor B: Tres tratamientos de control al mildiu [T1 = Testigo, sin aplicación; T2 = Tratamiento con un bioinsumo mediante aspersión al suelo con una mochila Jacto, Argentina de $20 \mathrm{~L}$ al momento de la siembra (tricobal a una dosis de $1 \mathrm{~L} / 25 \mathrm{~L}$ de $\mathrm{H}_{2} \mathrm{O}$ potable) y T3 = Estrategia de control químico mediante aspersión con una mochila Jacto, Argentina de $20 \mathrm{~L}$ (Metalaxyl a una dosis de 10g/5 L y Cimoxanil a una dosis de 20 g/7 L)]. El pesaje de los fungicidas se realizó con una balanza Kern KB, modelo Kb 600-2, Alemana. Se aplicó el Metalaxyl (sistémico) intercalado con el Cimoxanil (contacto) en tres oportunidades cada 15 días, tal como lo recomiendan Navia et al (1996), Fernandez-Northcote et al (1999). En cada aplicación se utilizó un adherente químico, para evitar el lavado por la lluvia. Las parcelas principales fueron los tratamientos para el control del mildiu ( $P$. farinosa Willd.) y la sub-parcela lo constituyeron las 36 accesiones de quinua.

El Tricobal es un producto conjugado de cepas nativas de Trichoderma harzianum, $T$. koningiopsis, Basillus subtilis y $B$. amyloliquefaciens, aisladas de diferentes zonas de Bolivia en los laboratorios de microorganismos de la Fundación PROINPA y actúa como protector de enfermedades de suelo, promotor de crecimiento, activa la resistencia natural de la planta y el enraizamiento (Ortuño et al 2010).

Las unidades experimentales estuvieron constituidas por un surco de $2 \mathrm{~m}$ de longitud con 20 plantas a $0.20 \mathrm{~m}$. Cada unidad experimental tuvo una superficie de $28.8 \mathrm{~m}^{2}$. En cada unidad experimental se marbeteó tres plantas seleccionadas al azar de cada surco central, al 50\% de botón floral al inicio de panojamiento y a 0.20 m de altura de planta.

La semilla que se empleó correspondió a una densidad de $8 \mathrm{~kg} \mathrm{ha}^{-1}$ en surcos y a chorro continuo. Esta fue distribuida a una profundidad de siembra de 2 a $3 \mathrm{~cm}$. La siembra fue realizada desde las 08:00 a.m. hasta las 11:00 a.m. para evitar la evapotranspiración de la humedad del suelo.

Se realizó el raleo de plantas (práctica que es recomendada para evitar la competencia entre plantas) a una distancia entre 0.10 a $0.12 \mathrm{~m}$ cuando las plantas alcanzaron $0.20 \mathrm{~m}$ de altura de planta. Se hizo tres deshierbes (el primero al 50\% 
de emergencia de las plantas, el segundo cuando las plantas tenían $0.20 \mathrm{~m}$ de altura y el tercero en el estado de botón floral o inicio de panojamiento) y dos aporques antes de la floración. Se aplicó urea (con $46 \%$ de $\mathrm{N}$, peruano, con fecha de vencimiento 2012) al primer aporque en una dosis de $350 \mathrm{~kg} \mathrm{ha}^{-1}$ y se eliminó las plantas atípicas (son plantas que no se parecen al fenotipo del cultivar) en cada surco durante la floración.

La cosecha, el trillado y venteo se realizó cuando el grano alcanzó la madurez fisiológica. El secado de panojas se hizo hasta alcanzar $12 \%$ de humedad. Luego los glomérulos fueron guardados en sobres manila de $25 \times 40 \mathrm{~cm}$, registrando el nombre del cultivar, el tratamiento y la fecha de cosecha. El trillado se realizó manualmente a mano, frotando las panojas durante el tiempo que fuera necesario para que los granos se separen de la broza. Finalmente, se efectuó el venteo durante 10 a 15 min con una ventiladora eléctrica Phillips, holandesa para eliminar todo tipo de impurezas presentes en las semillas, para luego ser almacenadas a temperatura de $5^{\circ} \mathrm{C}$ y $\mathrm{HR}$ de $5 \%$.

Variables de respuesta. Fueron consideradas las siguientes variables de respuesta:

Severidad de infección. Para la evaluación de la severidad de infección del mildiu (Peronospora farinosa) en campo, se evaluó el porcentaje de área foliar afectada (Danielsen \& Ames 2000) en tres hojas obtenidas aleatoriamente del tercio medio superior de plantas.

Para el análisis de la resistencia de las accesiones, se determinó El Area Bajo La Curva de Progreso de $P$. farinosa total en porcentaje - días
(ABCPPF) con la fórmula desarrollada por Shanner \& Finney (1977). El valor de ABCPPF se estimó en base a la severidad. El ABCPPF es una expresión o medida de avance de la enfermedad o incremento de daño en el tiempo (porcentaje-días). Para que los cultivares sean estadísticamente comparables, se calculó el Area Bajo la Curva de Progreso de $P$. farinosa relativa (ABCPPFrel), dividiendo el ABCPPF total entre el área máxima alcanzada (Campbell \& Maden 1990, Bonierbale et al 2008).

Rendimiento. Se evaluó el peso de los granos por tratamiento de control, accesión y repetición; y luego se transformó a $t$ ha $^{-1}$, según la longitud del surco y la distancia entre surcos contiguos.

Variables de caracterización. Se utilizó los descriptores de quinua de Rojas \& Pinto (2004). Para esto se consideró 10 variables cuantitativas (Tabla 1): Porcentaje de floración (días) (\%FL), madurez fisiológica (días) (FMF), longitud de planta (cm) (AAP), longitud de panoja (cm) (ILO), diámetro de tallo (mm) (TDM), diámetro de panoja (cm) (IDE), peso de 100 semillas (g) (GPE), diámetro de grano (mm) (GDI), espesor de grano (GFO) (mm) y porcentaje de emergencia (\%E).

Los datos de cada una de las variables de respuesta fueron analizados, previa comprobación de los supuestos de normalidad (cuando la distribución no se aproximó a la normal, se realizaron transformaciones) y homogeneidad de varianzas, de acuerdo al modelo estadístico planteado (Martínez-Garza 1988). 
Sobre la base del modelo definido se realizaron análisis de varianza para probar hipótesis acerca de los efectos fijos y comparaciones de medias mediante contrastes de un grado de libertad para determinar los cultivares resistentes y de alto rendimiento, así como el método de control para estas variables de respuesta. El análisis de varianza también sirvió para estimar los componentes de varianza para los efectos aleatorios. Para los análisis se usó el Proc GLM de SAS (SAS 2004).

Se realizó un análisis estadístico descriptivo de las variables cuantitativas para analizar el comportamiento de los diferentes cultivares con relación a cada carácter cuantitativo, determinando el promedio, el rango de variación, la desviación estándar y el coeficiente de variación que representan las medidas de tendencia central y de dispersión (Steel \& Torrie 1988).

Finalmente se hizo un análisis de correlación simple para conocer el grado de asociación existente entre las diferentes variables sin afectar la relación presente entre unidades $(\mathrm{cm}, \mathrm{mm}, \mathrm{g}$ y días). Para este análisis se utilizó el coeficiente de Pearson que se recomienda para datos de tipo multiestratos cuantitativos (Franco \& Hidalgo 2003) como es el caso del presente estudio.

\section{Resultados}

\section{Análisis de la resistencia al mildiu.}

El análisis de varianza (Tabla 1), mostró diferencias visibles al $1 \%$ de probabilidad $(\operatorname{Pr}<0.01)$ para bloques (blo), tratamientos (trat), la interacción blo * trat, cultivar (cult) y la interacción trat * cult. El análisis mostró un C.V. de $21 \%$ y un $\mathrm{R}^{2}$ de $91 \%$, indicando que el diseño experimental utilizado fue el apropiado para el control de las variaciones existentes. Estos análisis de probabilidad realizados indicaron que las severidades observadas en al menos uno de los tratamientos y en al menos uno de los cultivares fueron diferentes. Las interacciones significativas al $1 \%$ de probabilidad indicaron que los efectos de blo y trat no son independientes, de la misma manera ocurrió con los efectos de trat y cult que tampoco fueron independientes.

Tabla 1 Análisis de varianza para 36 accesiones de quinua (Chenopodium quinoa Willd.) evaluados bajo dos tipos de control al mildiu (Peronospora farinosa Willd.). El Paso, Cochabamba, 2011.

\begin{tabular}{|c|c|c|c|}
\hline FV & gl & SC & $\mathrm{CM}$ \\
\hline Total & 288 & 10.22 & \\
\hline Bloque (blo) & 3 & 0.91 & $0.30 * *$ \\
\hline Trat & 2 & 4.91 & $2.45^{* *}$ \\
\hline blo * trat & 4 & 0.48 & $0.12^{* *}$ \\
\hline Cultivar (cult) & 35 & 1.32 & $0.04 * *$ \\
\hline trat $*$ cult & 46 & 0.67 & $0.01 * *$ \\
\hline Error & 198 & 0.89 & 0.004 \\
\hline C.V. & 21.19 & & \\
\hline $\mathrm{R}^{2}$ & 0.91 & & \\
\hline
\end{tabular}

FV: Fuente de Variación, gl: grados de libertad, SC: Suma de Cuadrados Medios,

CM: Cuadrados medios, **: Notable al Pr<0.01 de probabilidad.

La comparación de medias entre T1, T2 y T3 (Figura 1), mostró diferencias manifiestas 1\% de probabilidad entre los tres tratamientos. El más afectado hasta el nivel letal fue el T1 (testigo) y el menos afectado fue el T3 (estrategia química). 


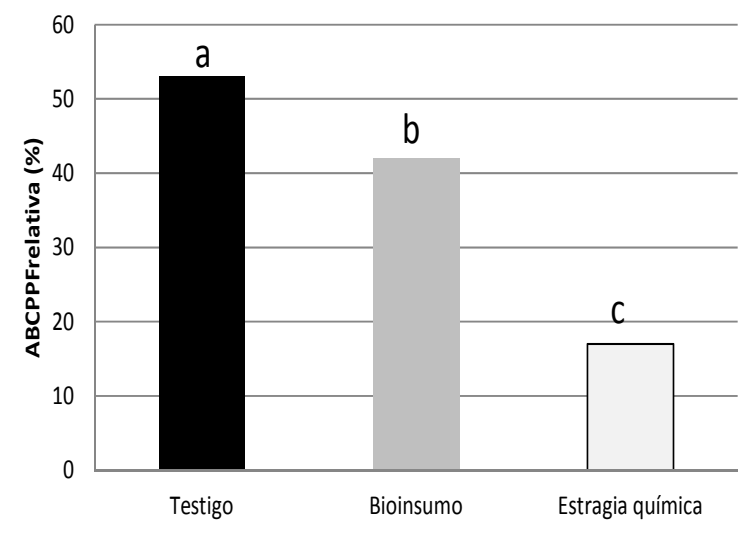

Figura 1 ABCPPFrel para mildiu ( $P$. farinosa Willd.) en 36 accesiones de quinua bajo tres tratamientos. Valores con la misma letra no son notablemente diferentes al $1 \%$ de probabilidad. Testigo, sin ninguna aplicación. Bioinsumo, aplicación con tricobal a la siembra. Estrategia química, Aplicación de fungicida sistémico y de contacto en al menos tres oportunidades.

Por otra parte comparando las medias del ABCPPFrel de los cultivares mediante la prueba de tukey al $1 \%$ de probabilidad (Figura 2), se observó diferencias notables para el ABCPPFrel, donde el cultivar 05Tardía fue el más susceptible (ABCPPFrel $=46 \%)$. Así mismo, los cultivares 01Tardía, 08Tardía, 12Tardía, 04Tardía, 11Tardía 10Tardía, 19Tardía y 18Tardía fueron susceptibles $($ ABCPPFrel $=39$ a 42\%). En cambio, los cultivares H172, A26, A03, A16, A22, A14 y H171 fueron los más resistentes $($ ABCPPFrel $=39$ a 42\%). De igual manera se observó una amplia variabilidad de resistencia parcial en todas las demás accesiones (ABCPPFrel $=20$ a 38\%).

\section{Análisis del rendimiento}

Se debe mencionar que para el análisis de rendimiento no se consideró el T1 (sin aplicación), debido a que todas las plantas de los cultivares evaluados murieron y no se logró cosechar ni un solo grano.

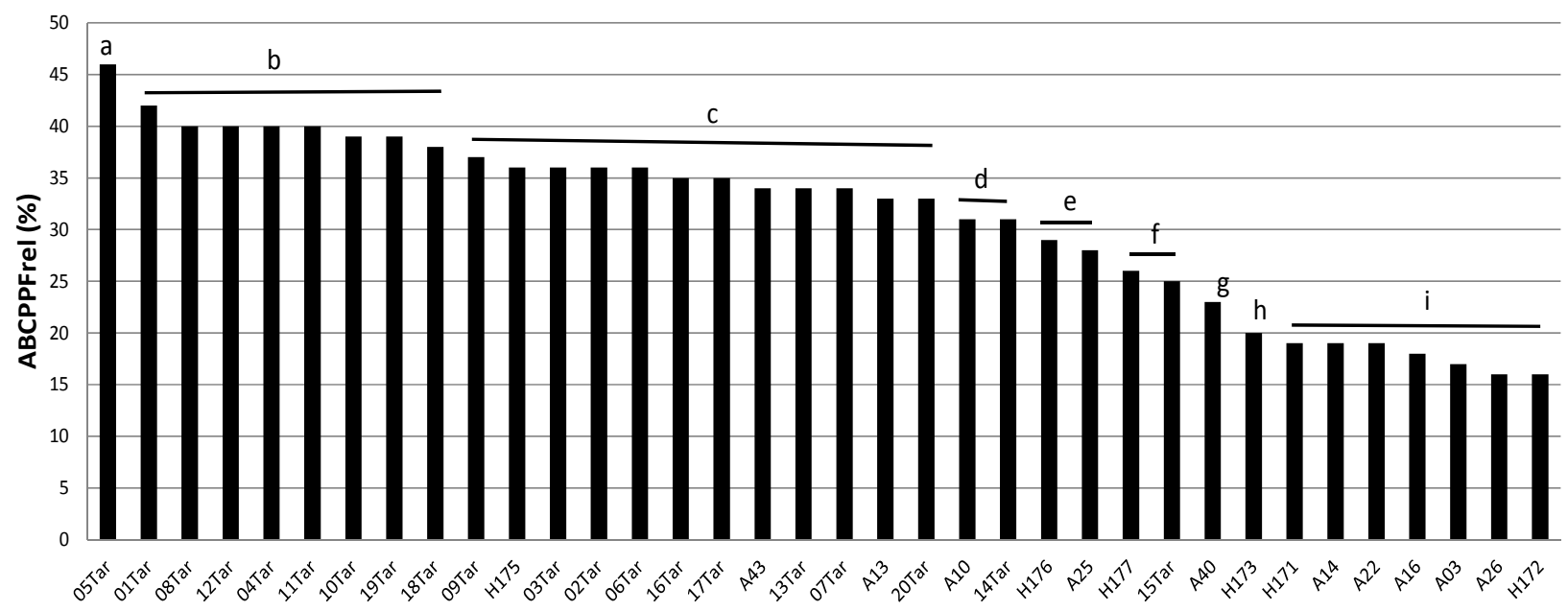

Figura 2 ABCPPFrel en 36 accesiones de quinua (Ch. quinoa Willd.) de valle y altiplano. El Paso, 2011.Valores con la misma letra no son notablemente diferentes al $1 \%$ de probabilidad. 
El análisis de varianza (Tabla 2) para porcentaje de emergencia (PE), rendimiento (Y) y peso de 100 semillas (P100sem), mostró C.V. de 19.38\%, $17.79 \%$ y $19.29 \%$; y valores de $\mathrm{R}^{2}$ de $0.41,0.61$ y 0.70 respectivamente. Esto sugirió que el diseño experimental utilizado fue apropiado para controlar la variación existente. Así mismo, se observó diferencias probadas al $1 \%$ de probabilidad para trat y cult en la variable $\mathrm{Y}, \mathrm{y}$ para cult y la interacción trat*cult en la variable P100. La interacción trat * cult, indicó que ambos factores nos son independientes.

Aun cuando no se observó diferencias obvias en el análisis de varianza del PE fue conveniente realizar una comparación múltiple de medias (Figura 3) la cual mostró diferencias visibles al $1 \%$ de probabilidad, observándose dos grupos claramente diferenciados, en uno se mostró un PE que varía entre 15 a 30\%, y en el otro grupo varío entre 31 a $60 \%$.

Tabla 2 Análisis de varianza para y porcentaje de emergencia (PE), rendimiento $(\mathrm{Y})$, peso de 100 semillas (P100sem) de 36 accesiones de quinua (Ch. quinoa Willd.) evaluados bajo dos tipos de control de mildiu ( $P$. farinosa Willd.). El Paso, Cochabamba, 2011.

\begin{tabular}{lcccc}
\hline \multicolumn{1}{c}{ FV } & gl & \multicolumn{3}{c}{ Cuadrados medios } \\
\hline & & PE & Y & P100sem \\
\cline { 3 - 5 } Total & & & & \\
Bloque & 3 & 9.36 & 3.38 & 1698338.07 \\
Tratamiento & 1 & 1.65 & $1.12^{* *}$ & 1272360.96 \\
(trat) & & & & \\
Cultivar (cult) & 35 & 8.14 & $1.12^{* *}$ & $6693143.25^{* *}$ \\
trat * cult & 35 & 6.24 & 2.61 & $1837434.14^{* *}$ \\
\hline Error & 185 & 37.37 & 7.45 & 5034196.71 \\
C.V. & & 19.38 & 17.79 & 17.29 \\
$\mathrm{R}^{2}$ & & 0.41 & 0.61 & 0.70 \\
\hline
\end{tabular}

FV: Fuente de Variación, gl: grados de libertad, PE: Porcentaje de emergencia (\%), Y: Rendimiento $\left(\mathrm{t}^{*} \mathrm{ha}^{-1}\right)$, P100sem: Peso de 100 semillas, **: Notable al $\operatorname{Pr}<0.01$ de probabilidad, C.V.; Coeficiente de Variación, $\mathrm{R}^{2}$ : Coeficiente de determinación.

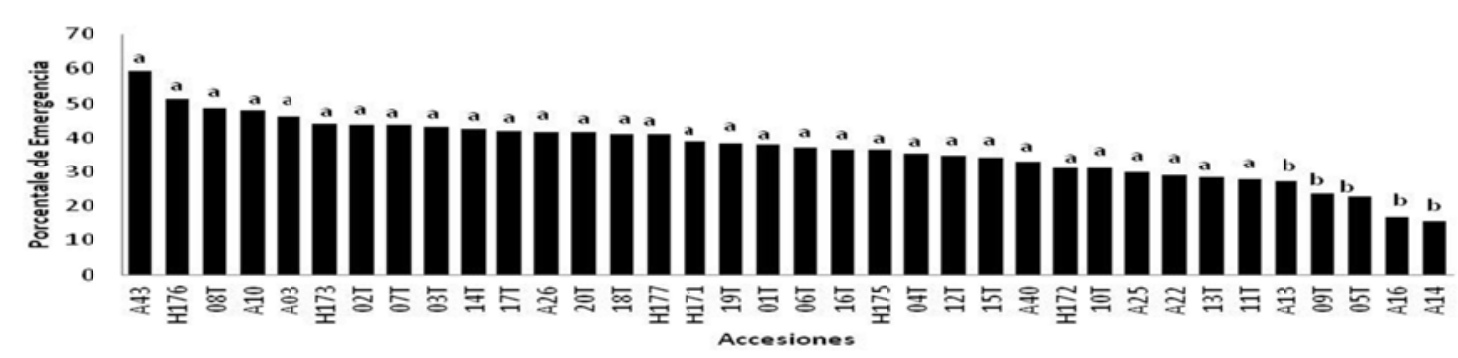

Figura 3 Porcentaje de emergencia (PE) en 36 accesiones de quinua (Ch. quinoa Willd.). El Paso, 2011.

El análisis de medias para $\mathrm{Y}\left(\mathrm{t} \mathrm{ha}{ }^{-1}\right)$ (Figura 4) mostró diferencias notables al $1 \%$ de probabilidad estadística entre los cultivares A40, H177, A26, H172, A25, A10,H176 y A03 que tuvieron los rendimientos más altos $\left(\mathrm{Y}=3.4\right.$ a $\left.6.34 \mathrm{t} \mathrm{ha}^{-1}\right)$. El otro grupo mostró rendimientos moderados a bajos $\left(\mathrm{Y}=0.74\right.$ a $\left.3 \mathrm{t} \mathrm{ha}^{-1}\right)$ y no hubo diferencias palpables entre ellos.
La comparación de las medias de rendimiento $(\mathrm{Y})$ para el T2 (bioinsumo) y T3 (estrategia de control químico) (Figura 5) mostró diferencias notorias dentro de cada cultivar, en tanto algunos cultivares como H171, H176, 14 Tardía, 03 Tardía y 15 Tardía no mostraron diferencias en Y. Otros cultivares como A03, 18Tardía, A22, H171 y A16 
se comportaron mejor en $\mathrm{Y}$ con el bioinsumo que con la estrategia química.

Respecto al peso de 100 semillas, la comparación de medias (Figura 6) mostró diferencias visibles al 1\% de probabilidad, denotándose tres grupos claramente diferenciados. Un primer grupo compuesto por los cultivares 05Tardía, 09Tardía, 08Tardía, 01Tardía, 02Tardía, 04Tardía y 07 Tardía mostraron un P100sem entre 34 a 35 g. Un segundo grupo mostró un P100sem entre 20 a 33 g y finalmente un tercer grupo mostró un P100sem entre 25 a 32 g. En el trabajo de campo se observó que el peso de los granos (g) fue influenciado directamente por el desarrollo del follaje, es decir, a mayor follaje menor tamaño y peso de grano, esto indicó que bajó la calidad del grano.

Se observó que las accesiones 13Tar, A14, A13, A22 y A10 fueron las que tuvieron mayor P100sem con la aplicación del fungicida (Figura 7). En cambio las accesiones 5Tar, 08Tar, 09Tar y H12, tuvieron P100sem más bajos con la aplicación de bioinsumo.

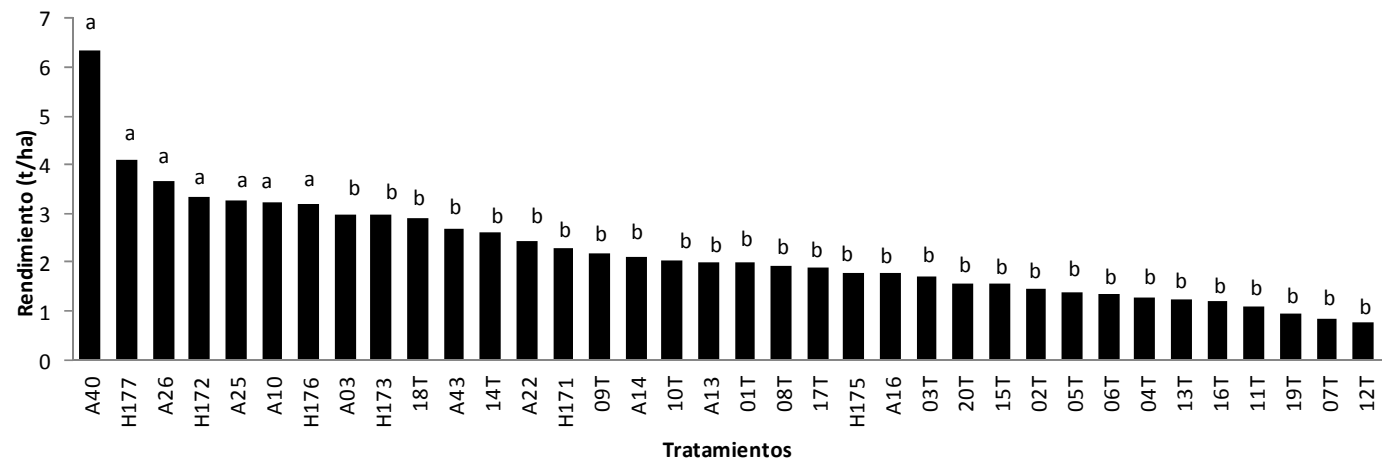

Figura 4 Rendimiento de grano (t ha ${ }^{-1}$ ) de 36 accesiones de quinua (Ch. quinoa Willd.). El Paso, 2011.

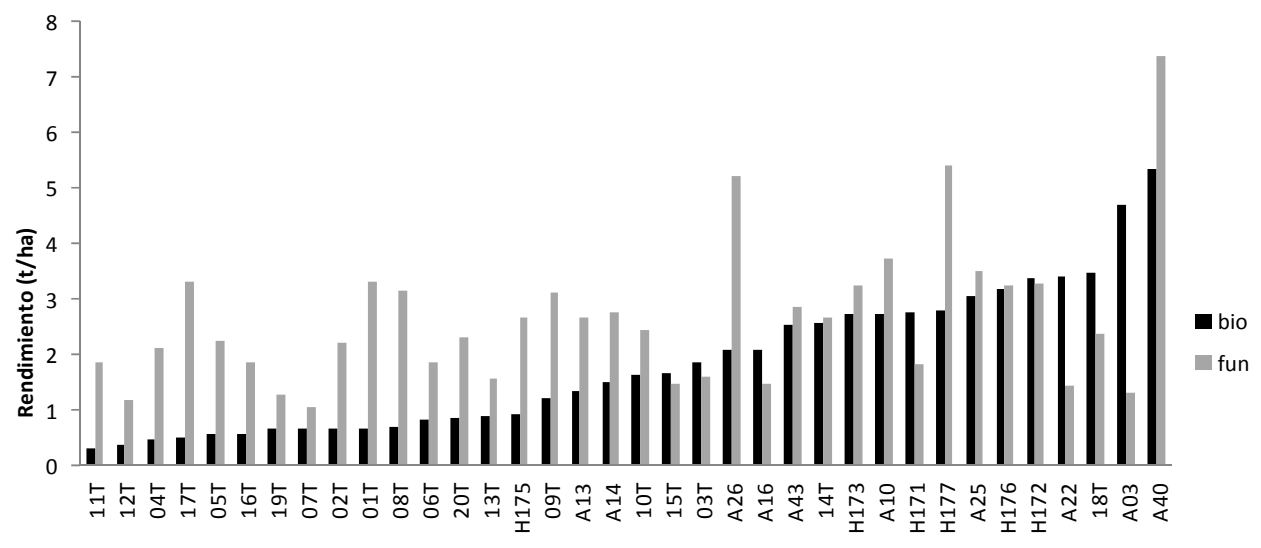

Figura 5 Rendimiento ( $\left.\mathrm{tha}^{-1}\right)$ de 36 accesiones de quinua (Ch. quinoa Willd.) con la aplicación de dos tipos de control de mildiu (P. farinosa Willd.). El Paso, Cochabamba, 2011. 


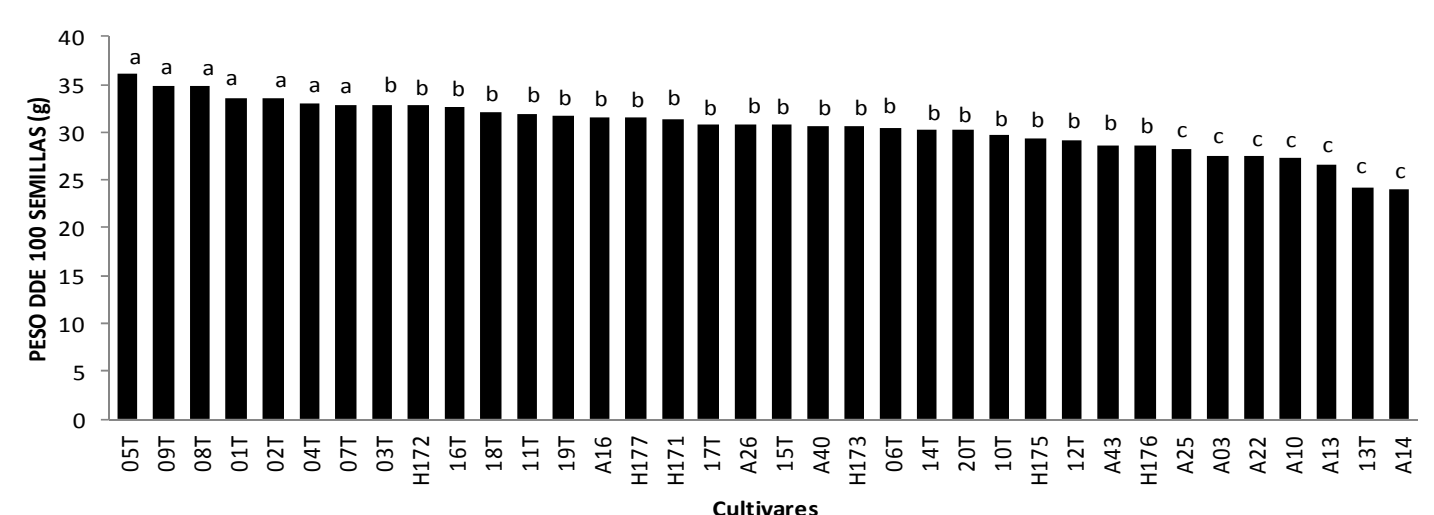

Figura 6 Peso de 100 semillas (g) de 36 accesiones de quinua (Ch. quinoa Willd.). El Paso, 2011.

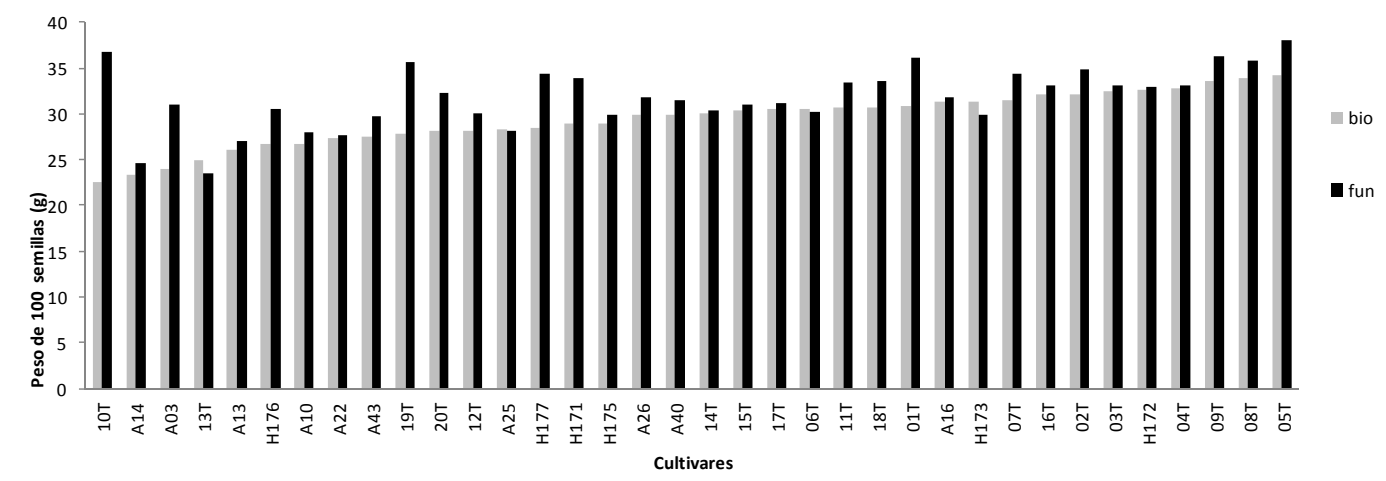

Figura 7 Peso de 100 semillas (gr) de 36 accesiones de quinua (Ch. quinoa Willd.) con la aplicación de dos tipos de control de mildiu (P. farinosa Willd.). El Paso, 2011

En la Tabla 3 se describen las variables, sus rangos de variación, el promedio, la desviación estándar (S) y el Coeficiente de Variación (CV). Este análisis mostro la amplia variabilidad existente entre las accesiones para cada una de las variables evaluadas. Fue notorio observar que todas las variables tuvieron un de CV (1.89 a 21\%) y estuvieron en el rango permitido para este tipo de investigación (Little \& Hills 1991).
El análisis realizado (Tabla 3), mostró que para el F\%L (días), el tiempo promedio desde la siembra, fue de 87.96 días, con un CV de 1.89\%. El rango de variación fue de 84.2 días para las más precoces y 93.60 días para las más tardías. Las líneas más precoces fueron procedentes del Altiplano del departamento de La Paz (11Tardía, 12Tardía, y 05Tardía). Las más tardías procedieron del Valle Alto de Cochabamba (A13 y A16). 
Tabla 3 Parámetros de estadística descriptiva de 11 variables cuantitativas caracterizadas y evaluadas en los cultivares de quinua (Ch. quinoa Willd.). El Paso, Cochabamba, 2011.

\begin{tabular}{llcccc}
\hline \multicolumn{1}{c}{ Variables } & \multicolumn{1}{c}{ Cód. } & $\begin{array}{c}\text { Rango de } \\
\text { Variación }\end{array}$ & Promedio & S & CV (\%) \\
\hline 50 \% de floración (días) fenológico & \%FL & $84.2-93.60$ & 87.97 & 2.36 & 1.89 \\
Madurez fisiológica (días) fenológico & FMF & $120.3-132$ & 125.28 & 33.02 & 4.91 \\
Longitud de planta (cm) morfológico & AAP & $119.3-171$ & 145 & 13.49 & 21.38 \\
Longitud de panoja (cm) morfológico & ILO & $13.67-65.33$ & 39.43 & 8.61 & 21.84 \\
Diámetro de tallo (mm) morfológico & TDM & $9.3-11.5$ & 103.125 & 5.78 & 11.99 \\
Diámetro de panoja (cm) morfológico & IDE & $33.2-81$ & 45.744 & 7.14 & 17.06 \\
Peso de 100 semillas (g) grano & GPE & $0.23-0.36$ & 0.30 & 5.14 & 17.28 \\
Diámetro de grano (mm) grano & GDI & $1.9-3$ & 2.426 & 0.18 & 7.53 \\
Espesor de grano (mm) grano & GFO & $0.9-1$ & 1.062 & 0.09 & 8.56 \\
Porcentaje de emergencia & \% E & $15.67-59.62$ & 36.96 & - & 19.38 \\
\hline
\end{tabular}

S: Desviación estándar, C.V.: Coeficiente de variación.

Para FMF (días), se observó que las líneas llegaron a la madurez fisiológica en 125 días (Tabla 3), el coeficiente de variación fue de 4.91\%, Las líneas más precoces (07Tardía, 02Tardía y 08Tardía) alcanzaron la madurez fisiológica a los 120 días. Estas accesiones provinieron del Altiplano del departamento de La Paz. Las líneas más tardías (132 días) provinieron de los Valles de Cochabamba (A03 y A16), destacándose claramente que las más precoces fueron precedentes del Altiplano. Aparentemente la precocidad observada fue debida a factores ambientales como el fotoperiodo (8 horas luz).

Se debe mencionar que las líneas procedentes de altura probablemente son precoces porque adquieren características propias para sobrevivir y escapar de las condiciones áridas del altiplano y mantener de esta forma la especie, ocurre lo contrario en líneas procedentes de Valle, donde las condiciones son favorables y estimulan la prolongación de sus fases fenológicas (Bonifacio \& Saravia 1999).
En referencia a la AAP $(\mathrm{cm})$, se observó un promedio de $145 \mathrm{~cm}$, con un coeficiente de variación de $21.38 \%$, asimismo el rango de variación fue de 119 a 171 cm. Las líneas más pequeñas (12Tardía, 05Tardía y 06Tardía) vinieron del Altiplano de La Paz. Las líneas de mayor altura (A14, A03 y A22) procedieron del Valle Alto de Cochabamba. Estas diferencias podrían atribuirse posiblemente a las características genéticas propias de cada línea, pero también probablemente las líneas han expresado de diferente manera su crecimiento por efecto del mildiu (Bonifacio 1997).

Respecto a la ILO (cm), se estimó un promedio de $39.43 \mathrm{~cm}$ y un CV de 21.84\%, debido a la baja dispersión existente respecto al promedio. El rango de variación fue de $13.67 \mathrm{~cm}$ para las líneas de panojas de cortas como la 12Tardía, 05Tardía y 06Tardía y las líneas de panojas largas $(65.33$ cm de longitud) como en las líneas A14, A3 y A22 de los Valles. Estas últimas líneas podrían ser útiles para incrementar el rendimiento de la quinua, 
debido a que este carácter es un componente importante del rendimiento.

En referencia al TDM (mm) el promedio fue de $10.3 \mathrm{~mm}$ y el CV de $11.99 \%$ (Tabla 3), con un rango de variación de 9.3 a 11.52 mm. Diámetros menores se presentaron en líneas procedentes del Altiplano (06Tardía, 12Tardía y 05Tardía). Por el contrario, diámetros mayores se observaron en las líneas de Valle (A14, A03 y A22).

Por lo mencionado al parecer en el desarrollo del diámetro del tallo influye el genotipo, el ciclo fenológico y las características climáticas de cada región (Revollo 2004).

El IDE (cm) fue de $5 \mathrm{~cm}$, con un CV de $17.06 \%$ y un rango entre 33 y $81 \mathrm{~cm}$. Las líneas con panojas de menor diámetro (06Tardía, 05Tardía y 12Tardía) procedieron del Altiplano, Por el contrario diámetros mayores se registraron en accesiones procedentes del Valle Alto de Cochabamba (A14, A03, Y A22). El menor diámetro probablemente se atribuye tanto a las condiciones ambientales de la zona del Valle cuyas temperaturas son más elevadas en los meses de noviembre a febrero que en el altiplano.

Acerca del GPE (g), se observó un promedio de 0.80 g, un CV de $14,88 \%$ y un rango de variación entre 0.7 a 0.91 g. Se identificó pesos bajos de 100 semillas en las líneas de Valle (H175, H176, A43). En cambio las líneas provenientes del departamento de La Paz (19Tardia, 10Tardia, y
07Tardia) mostraron mayor peso de semillas. Esta característica al parecer se encuentra relacionada con el diámetro y espesor de grano, de manera que líneas que desarrollen mayor tamaño de semilla y peso, tendrán una buena ventaja en el mercado.

En referencia al IDE (mm) se observó un promedio de $2.42 \mathrm{~mm}$, un CV de $7.53 \%$ y un rango de variación entre 1,9 a $3 \mathrm{~mm}$ entre las líneas. Líneas A10 y 13Tardía tuvieron los granos de diámetro pequeño. Las líneas 07Tardía, 14Tardía, 01Tardía y 19Tardía tuvieron el mayor diámetro, o mayor tamaño de grano (Tabla 3).

En cuanto al GFO (mm), se observó un promedio de $1,06 \mathrm{~mm}$, un CV de $8.56 \%$ y un rango de variación de 0,9 a $1 \mathrm{~mm}$, entre las líneas de menor y mayor espesor de grano. Las líneas A14, H172, y 13Tardía mostraron menor espesor de grano. Las líneas A25, H177 y 18Tardía tuvieron mayor espesor de grano. Esta variable se relaciona estrechamente con el diámetro de grano y caracterizan al tamaño de grano.

Finalmente el \%E mostró un promedio de 36.96\%, un CV de 19,38 \% y un rango de variación de 15,67 a $59.62 \%$.

La Tabla 4, muestra una matriz con las correlaciones de Pearson entre variables cuantitativas. Se han considerado para el análisis solamente las correlaciones altas $>0.50$ y que estas sean significativas. 
Tabla 4 Correlaciones de Pearson entre variables cuantitativas.

\begin{tabular}{|c|c|c|c|c|c|c|c|c|c|c|c|}
\hline & FL & FMF & AAP & ILO & TDM & IDE & GPE & $\mathrm{Y}$ & GDI & GFO & ABCPPFR \\
\hline & 1 & & & & & & & & & & \\
\hline FL & 0.35 & 1 & & & & & & & & & \\
\hline FMF & & & & & & & & & & & \\
\hline & $0.52^{* *}$ & 0.41 & 1 & & & & & & & & \\
\hline AAP & & & & & & & & & & & \\
\hline ILO & 0.43 & 0.30 & $0.65 * *$ & 1 & & & & & & & \\
\hline & $0.55^{* *}$ & $0.57 * *$ & $0.97 * *$ & & 1 & & & & & & \\
\hline TDM & 0.14 & 0.37 & $0.60 * *$ & $\begin{array}{r}\mathbf{0 . 7 4} * * \\
0.47\end{array}$ & $0.62 * *$ & 1 & & & & & \\
\hline IDE & -0.18 & -0.32 & - 0.32 & -0.17 & -0.35 & -0.47 & 1 & & & & \\
\hline GPE & 0.26 & 0.28 & $0.59 * *$ & 0.33 & $0.57 * *$ & $0.50 * *$ & -0.16 & 1 & & & \\
\hline GDI & 0.12 & -0.03 & -0.20 & -0.10 & -0.18 & -0.26 & 0.26 & -0.30 & 1 & & \\
\hline GFO & -0.01 & -0.43 & -0.10 & -0.07 & -0.17 & -0.27 & $0.57 * *$ & -0.01 & 0.03 & 1 & \\
\hline ABCPPFR & -0.41 & $\overline{-}$ & $-0.58^{* *}$ & -0.37 & $-0.63 * *$ & -0.48 & $0.50 * *$ & -0.38 & -0.12 & 0.45 & 1 \\
\hline
\end{tabular}

F\%L: 50 \% de floración (días), FMF: Madurez fisiológica (días), AAP: Longitud de planta (cm) ILO: Longitud de panoja (cm), TDM: Diámetro de tallo (mm), IDE: Diámetro de panoja (cm), Y: rendimiento, GPE: Peso de 100 semillas (g), GDI: Diámetro de grano (mm), GFO: Espesor de grano (mm), ABCPPFrel: Área de progreso del mildiu. *: Notablemente diferentes al 5\% de probabilidad, **: Notablemente diferentes al $1 \%$ de probabilidad.

La correlación más alta y positiva se dio entre las variables AAP y TDM ( $r=0.97)$. Otras variables altamente y positivamente correlacionadas con AAP fueron: ILO $(r=0.65)$, IDE $(r=0.60)$ e Y (r $=0.59)$. Por otra parte, existió una alta y negativa correlación entre AAP y ABCPPFrel $(r=-0.58)$.

En las accesiones evaluadas, las plantas con menor altura poseen panojas pequeñas y de menor diámetro, menor diámetro de tallo, menor rendimiento y mayor susceptibilidad al mildiu y del mismo modo, las plantas más altas tuvieron mayor longitud y diámetro de panoja, mayor diámetro de tallo, mayor rendimiento y resistencia al mildiu.

Por otra parte, fue notorio observar que la variable ABCPPFrel de mildiu se correlacionó negativamente con la variable fenológica FMF
( $r=-0.58)$, también con las variables morfológicas AAP $(r=-0.58)$ y TDM $(r=-0.63)$. Por otra parte, se correlacionó positivamente con la variable de grano GPE ( $\mathrm{r}=0.50)$, esto indicaría que hay una relación inversamente proporcional entre la resistencia al mildiu y las variables mencionadas. Esto significa que las accesiones resistentes a mildiu fueron tardías, altas, con tallos gruesos y con menor peso de 100 semillas. Estas características pertenecen a las accesiones de Valle. Por otra parte, las accesiones susceptibles serían aquellas precoces, con tallos delgados, menor altura de planta y mayor peso de 100 semillas. Estas características son propias de las accesiones de Altiplano.

La correlación positiva entre el p100 con las variables TDM $(r=0,98$ e IDE $(r=0,40)$, permite 
deducir que plantas con tallos y panojas más gruesos tuvieron mayor peso de granos.

La correlación positiva entre el GPE con las variables GFO $(\mathrm{r}=0,57)$, permite deducir que cuando mayor sea el peso de 100 semillas, los granos serán de mayor espesor.

La correlación positiva de $\mathrm{Y}$ con las variables AAP $(r=0,59), \operatorname{TDM}(r=0,57)$ e IDE $(r=0,50)$, indica que las plantas altas con panojas y tallos gruesos tienden a desarrollar mayores rendimientos.

\section{Discusión}

En nuestro estudio hemos observado que los cultivares tardíos fueron altamente resistentes al mildiu y los cultivares precoces fueron susceptibles, esto confirmó lo observado por Bonifacio y Saravia (1999). También encontramos que los cultivares de Valle fueron altamente resistentes, contrariamente los cultivares de Altiplano que fueron susceptibles, confirmando los resultados encontrados por Danielsen \& Ames (2000). Se debe destacar que en el estudio se encontró una amplia variabilidad en la resistencia al mildiu, indicando esto una complejidad en la que probablemente están involucrados genes mayores, menores y genes $\mathrm{R}$ vencidos (Parlevliet 1997, Gabriel et al 2007). Al respecto Alandia et al (1979), encontraron un rango amplio de susceptibilidad frente al mildiu en condiciones de la Estación Experimental de Patacamaya en Bolivia hallando infecciones sistémicas, lesiones foliares casi completas y hasta alto grado de resistencia. Bonifacio (1997), menciona que estos hechos se explican en términos de evolución y 40 domesticación de la especie, puesto que durante este proceso, las condiciones del medio ambiente era favorable para la presencia del mildiu donde sólo los tipos de quinua con mayor resistencia han sobrevivido y han perdurado en el tiempo.

Una contribución importante de nuestro trabajo fue el hecho de encontrar cultivares resistentes que tuvieron igual comportamiento frente a $P$. farinosa (mildiu) cuando fueron tratados con tricobal y con la estrategia de control químico, que se reflejó en el rendimiento. Esto está indicando que algunos cultivares resistentes tuvieron rendimientos moderados a altos aplicando el tricobal (bioinsumo), aunque esto no fue una regla general.

Fue notorio también observar que los cultivares resistentes tuvieron menor diámetro (tamaño de semilla), confirmando lo mencionado por Bonifacio (1997), que al evaluar líneas resistentes al mildiu durante dos ciclos de selección, identificó material con buen nivel de resistencia al mildiu, pero asociada al ciclo tardío y al tamaño de grano pequeño, lo que es poco favorable para el altiplano y escasamente preferidos en el mercado.

La alta correlación negativa y significativa entre el ABCPPrel y las variables FMF: Madurez fisiológica (días), AAP: Longitud de planta (cm) ILO: Longitud de panoja (cm), TDM: Diámetro de tallo (mm), IDE: Diámetro de panoja (cm) y GPE: Peso de 100 semillas (g), está indicando que estas variables son fuertemente afectadas por el mildiu, es decir cuando el ataque del mildiu es severo, afecta directamente a la FMF, a la AAP, a la ILO, al TDM y al IDE y al GPE. Por lo tanto afectan al rendimiento. Esto concuerda con lo observado por diversos investigadores (Otazú et al 
1976, Bonifacio 1997, Bonifacio \& Saravia 1999, Danielsen \& Ames 2000, Ochoa et al 1999).

\section{Conflictos de interés}

Esta investigación recibió financiamiento total del Proyecto "Fortaleciendo capacidades de innovación participativa para luchar contra la pobreza rural” (IP - Holanda) y no presenta conflictos de interés.

\section{Agradecimientos}

Se agradece el apoyo económico del proyecto "Fortaleciendo capacidades de innovación participativa para luchar contra la pobreza rural” (IP - Holanda).

\section{Literatura citada}

Acevedo F. El cultivo de la caraota. Fondo editorial UNELLEZ. Ediciones de la Universidad Ezequiel Zamora. 2003;208 pp.

Aegerter BJ, Nuñez JJ, Davis RM. Detection and Management of Downy Mildew in Rose Rootstock. Plant Dis. 2002; 86(12):1363-1368.

Alandia S. Enfermedades. Páginas 137-144 in Quinua y kanihua. Cultivos Andinos. Tapia et al (ed.). IICA, Bogotá, Colombia. 1979.

Alfano JR, Collmer A. The type III (Hrp) secretion pathway of plant pathogenic bacteria: Trafficking harpins, Avr proteins, and death. J Bacteriol. 1997;179:56555662.

Bonierbale M, De Haan S, Forbes A. Procedures for standard evaluation trials of advanced potato clones. In: An international cooperators' guide. International Potato Center, Lima, Peru. 2008;124 pp.

Bonifacio A. Mejoramiento de la quinua para resistencia a factores adversos en Bolivia. Páginas 75-78 in Primer Taller de PREDUZA en resistencia duradera en cultivos altos en la zona andina. Daniel Danial (Ed.). Proyecto de Resistencia Duradera en la Zona Andina, PREDUZA. Quito, Ecuador. 1997.

Bonifacio A, Saravia R. Evaluación de la resistencia al mildiu en quinua. In: Proceedings of the Tercer Taller de Preduza en Resistencia Duradera en Cultivos Altos en la Zona Andina, 27-29 de Septiembre de 1999. Cochabamba, Bolivia. 1999;49-59 pp.

Campbell CL, Madden LV. Introduction to plant disease epidemiology. John Wiley \& Sons, NY, USA. 1990;532 pp.

Danielsen S, Ames T. El mildiu (Peronospora farinosa) de la quinua (Chenopodium quinoa) en la zona andina. Centro Internacional de la Papa, Lima, Peru. 2000;32 pp.

FAO (Food and Agriculture Organization of the United Nations). Under-utilized Andean food crops. Latin América and the Caribbean, Rome, Italy. 2011.

Fernandez-Northcote EN, Navia O, Gandarillas A. Bases de las estrategias de control químico del tizón tardío de la papa desarrolladas por PROINPA en Bolivia. 
Revista Latinoamericana de la Papa, Perú. 1999;11:1-25.

Franco T, Hidalgo R. Análisis Estadístico de datos de Caracterización morfológica de Recursos genéticos. Instituto internacional de recursos fitogenéticos Cali - Colombia. 2003; 89 pp.

Gabriel J, Coca A, Plata G, Parlevliet JE. Characterization of the resistance to Phytophthora infestans in local potato cultivars in Bolivia. Euphytica (Netherlands). 2007; 153:321-328.

Gabriel J. Documento marco: Estrategias y perspectivas del mejoramiento genético de papa (Solanum tuberosum L.) en Bolivia. ISBN: 978-99954-743-2-4, Fundación PROINPA, Cochabamba, Bolivia. 2010;60 pp.

Gandarillas H. Mejoramiento de la Quinua. En: La Quinua y la Kañiwa: Cultivos Andinos. M. Tapia (ed.). Centro Internacional de Investigación para el Desarrollo, Santafé de Bogotá. 1979;65-82 pp.

IBTA. Curso de Cultivos. Programa Andes Altos - IICA. IBTA / DNS. Catálogo de variedades mejoradas de quinua y uso de Semilla certificada. IBTA, Regional LP / DNS, oficina y laboratorio de semilla, La Paz, Bolivia. 1987;20 pp.

Little T \& Hills JF. Métodos estadísticos para

la investigación en agricultura. Trillas, México D.F., México. 1991;270 pp.

López J. La calidad de la proteína en la Quinua, (Chenopodium quinoa Willd). 42
Biblioteca del Centro Internacional de la Papa. Lima, Perú. 1976.

Martinez-Garza A. Diseños experimentales: Métodos y elementos de teoría. Editorial Trillas, México D.F., México. 1988;756 pp.

Mujica A. Parámetros genéticos e índices de selección en quinua (Chenopodium quinoa Willd.). Tesis de Doctorado. Colegio de Postgraduados, Centro de Genética. Montecillos, México. 1988;122 pp.

Muñoz L, Monteros C y Montesdeoca P. A cocinar con quinua. EE. Santa Catalina, INIAP. Quito, Ecuador. Publ. Miscl. 1990; 55: 7-120.

Navia O, Fernandez-Northcote EN. Estrategias para la integración de resistencia y control químico del tizón. Fitopatología, ficha técnica 3, PROINPA, Cochabamba, Bolivia. 1996.

Ochoa J, Danial D. Manejo de patógenos especializados en el mejoramiento genético de plantas para resistencia a enfermedades. In: Curso sobre aspectos técnicos en el manejo de los patosistemas de cultivos altos. 1999;17-31 pp.

Ochoa J, Frinking HD, Jacobs Th. Postulation of virulence groups and resistance factors in the quinoa/ downy mildew pathosystem using material from Ecuador. Plant Pathology. 1999;48:425-430.

Ortiz R. Resistencia a factores adversos y parasitoides controladores biológicos de "q’hona q'hona" (Eurysacca melanocampta Meyrick) en manejo integrado de plagas en el cultivo de quinua. 
Universidad Nacional del Altiplano. Puno, Perú. 1998;20 pp.

Ortuño N, Navia O, Meneces E, Barja D, Villca S. Catálogo de bioinsumos: para mejorar la productividad de los cultivos ecológicos y convencionales. Fundación PROINPA, Cochabamba, Bolivia. 2010;42 pp.

Otazú V, Aguilar PC, Canahua A. Resistencia en quinua (Chenopodium quinoa) al mildiú Peronospora effusa). Fitopatología. 1976;11:47-49.

Parlevliet JE. Resistencia durable a patógenos y como mejorar por este tipo de resistencia. In: Primer Taller de PREDUZA en resistencia duradera en cultivos altos en la zona andina. Daniel Danial (Ed.). Proyecto de Resistencia Duradera en la Zona Andina, PREDUZA. Quito, Ecuador. 1997;1-16 pp.

Revollo LM. Variabilidad genética de cuatrocientos veintiún poblaciones de quinua real conservadas en el banco nacional de granos altoandinos. Tesis de grado, Carrera de ingeniería agronómica, facultad de ciencias Naturales y Medio Ambiente, Universidad Loyola. La Paz, Bolivia. 2004;99 pp.

Rojas W, Pinto M, Bonifacio A, Gandarillas A. Banco de Germoplasma de Granos Andinos. In: W. Rojas, M. Pinto, JL. Soto, M. Jagger y S. Padulosi (eds). Granos Andinos: Avances, logros y experiencias desarrolladas en quinua, cañahua $\mathrm{y}$

amaranto en Bolivia. Bioversity
International, Roma, Italia. 2010;24-38 pp.

Rojas W. Análisis de la diversidad genética del germoplasma de quinua (Chenopodium quinoa Willd.) de Bolivia, mediante métodos multivariados. Tesis de Maestria, Universidad Austral de Chile, Facultad de Ciencias Agrarias. Valdivia - Chile.1998;209 pp.

Rojas W, Pinto M. Descriptores para quinua, Fundación para la Promoción e Investigación en Productos Andinos (PROINPA) La Paz, Bolivia.Rojas W, Cayoja M, Espíndola G. Catálogo de colección de quinua conservada en el banco nacional de granos altoandinos. Fundación PROINPA, La Paz, Bolivia. 2004; 129 pp.

SAS Institute Inc. SAS/STAT Users Guide, Version 9.2, Fourth Edition, Vol. 2, SAS Institute Inc., Cary, N.C. 2004.

Franco TL, Hidalgo R (eds.). Análisis Estadístico de Datos de Caracterización Morfológica de Recursos Fitogenéticos. Boletín técnico no. 8, Instituto Internacional deRecursos Fitogenéticos (IPGRI), Cali, Colombia. 2003;89 pp.

SENAMHI (Servicio Nacional de Meteorología e Hidrología). Datos climáticos (temperatura, precipitación y humedad relativa) de la comunidad de Letanías, población de Viacha provincia Ingavi, departamento de La Paz. 2010.

Shanner G, Finney RN. The effect of nitrogen fertilization on the expression of slowmildewing resistance in knox wheat. Phytopathology. 1977;67:1051-1056. 
Steel R, Torrie J. Bioestadística: principios y procedimientos. 2da edición. México:GrawHill. 1988. 622 pp.

Tapia M. Historia de distribución geográfica de la quinua in Cultivos Andinos: Quinua Cañihua. CIID-IICA. Bogotá. 1979;227 pp.
Tomma BPHJ. Alternaria spp.: from general saprophyte to specific parasite. Molecular Plant Pathology. 2003;4(4):225-236.

Viñas O. Exportación de quinua orgánica (Chenopodium quinoa Willd.) de la república de Bolivia. La Paz, Bolivia. 2000;14 pp. 